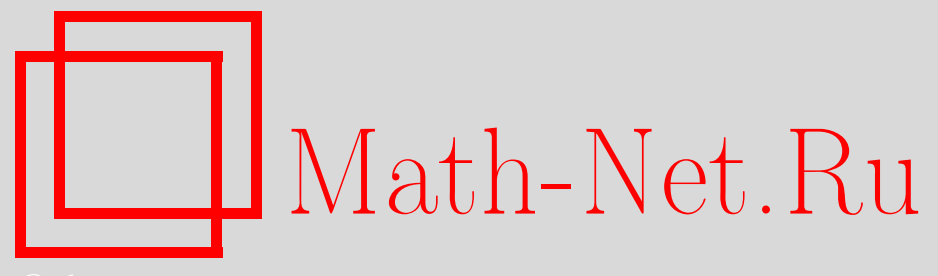

В. Йовович, Г. Килибарда, О числе функций алгебры логики в классах Поста $F_{8}^{\mu}$, Дискрет. матем., 1999, том 11 , выпуск 4, 127-138

DOI: https://doi.org/10.4213/dm398

Использование Общероссийского математического портала Math-Net.Ru подразумевает, что вы прочитали и согласны с пользовательским соглашением http://www. mathnet.ru/rus/agreement

Параметры загрузки:

IP: 44.207 .124 .84

26 апреля 2023 г., 13:48:29

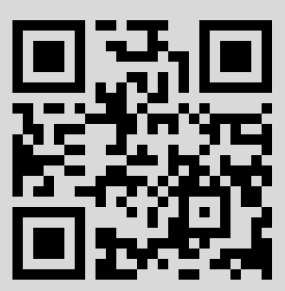




\title{
О числе функций алгебры логики в классах Поста $F_{8}^{\mu}$
}

\author{
(C) 1999 г. В. Йовович, Г. Килибарда
}

\begin{abstract}
Рассматривается проблема перечисления функций алгебры логики от $n$ переменных ранга $k$ из классов Поста $F_{8}^{\mu}$. Эта проблема, выраженная на языке теории множеств, эквивалентна проблеме перечисления всех $k$-семейств попарно различных подмножеств $n$-множества, у которых любые $\mu$ членов имеют непустое пересечение. Получены формулы в терминах теории графов для числа элементов этих классов. Явные формулы для случаев $\mu=2, k \leqslant 8$ (для $k \leqslant 6$ они приведены в конце настоящей статьи), $\mu=3,4, k \leqslant 6$, и при любом $n$ получены с помощью компьютера. В качестве следствия получены соответствующие результаты для классов $F_{5}^{\mu}$.
\end{abstract}

\section{1. Введение}

Классы Поста суть множества функций алгебры логики, которые замкнуты относительно операций суперпозиций. Пост явно описал структуру этих классов $[1,2]$. Функция алгебры логики $f$ от $n$ переменных имеет ранг $k$, если существует точно $k$ различных двоичных наборов длины $n$, на которых она принимает значение 1 . Если $\mathscr{P}$ - класс Поста, то множество всех функций от $n$ переменных ранга $k$ из этого класса обозначим через $[\mathscr{P}]_{k}^{(n)}$ и назовем $(n, k)$-сечением класса Поста $\mathscr{P}$.

Проблема определения мощности $(n, k)$-сечений для любых натуральных $n$ и $k$, $0 \leqslant k \leqslant 2^{n}$, для некоторых классов Поста еще не решена. Например, она не решена для класса $F_{8}^{\mu}$, который можно определить следующим способом. Функция алгебры логики $f$ принадлежит классу $F_{8}^{\mu}$ тогда и только тогда, когда любые $\mu$ наборов, на которых она обращается в единицу, имеют общую единичную компоненту (среди этих $\mu$ наборов могут быть и одинаковые). В настоящей статье рассматривается класс $F_{8}^{\mu}$ (а также и дуальный случай, класс $F_{4}^{\mu}$ ). В качестве следствия получены соответствующие результаты для классов $F_{5}^{\mu}$ и $F_{1}^{\mu}$.

Гиперграфы, у которых для любой пары их вершин существует ребро, содержащее только одну из них, но не обе одновременно, называются $T_{0}$-гиперграфами. Если на множестве ребер гиперграфа задан некоторый линейный порядок, то его вместе с этим линейным порядком назовем упорядоченным гиперграфом. Гиперграф, у которого любое множество вершин мощности, не большей $\mu$, является множеством смежных вершин, называется $\mu$-полным гиперграфом. Тогда проблему определения мощности $(n, k)$-сечения класса $F_{8}^{\mu}$ можно свести к проблеме перечисления $\mu$-полных упорядоченных $T_{0}$-гиперграфов, у которых $k$ вершин и $n$ ребер, а ее в 
свою очередь можно свести к проблеме определения числа всех гиперграфов с фиксированным числом вершин и ребер и с заданным числом независимых множеств вершин. В настоящей статье независимое множество вершин интерпретируется в терминах раскраски гиперграфа.

\section{2. Основные понятия}

Приведем некоторые понятия теории гиперграфов и теории функций алгебры логики, которые будут использованы в нашей работе.

Под гиперграфом мы понимаем пару $H=(V, \mathscr{E})$, где $V-$ конечное непустое множество, а $\mathscr{E}=\left(e_{\lambda}, \lambda \in \Lambda\right)-$ конечное семейство подмножеств множества $V$ (эти подмножества могут повторяться или быть пустыми). Семейство $\mathscr{E}$ может быть также пустым. Назовем элементы множества $V$ вершинами, а члены семейства $\mathscr{E}$ ребрами данного гиперграфа $H$. В последующем множество вершин гиперграфа $H$ будем обозначать также и через $V H$, а семейство его ребер через $\mathscr{E} H$. Если $|V|=n$ и $|\mathscr{E}|=m$, то гиперграф $H$ назовем $(n, m)$-гиперграфом. Если в этом определении вместо семейства $\mathscr{E}$ возьмем набор $\left(e_{1}, e_{2}, \ldots, e_{m}\right)$, где $e_{i} \subseteq V, i=1, \ldots, m$, то получим определение упорядоченного $(n, m)$-гиперграфа. Его ребрами являются множества $e_{i}, i=1, \ldots, m$.

Пару $H=(V, \mathscr{E})$, где $V-$ конечное непустое множество и $\mathscr{E} \subseteq 2^{V}$, назовем гиперграфом без кратных ребер (б.к.р. гиперграфом). Множество $V$ является множеством вершин, а $\mathscr{E}$ множеством ребер данного б.к.р. гиперграфа (в отличие от случая гиперграфа здесь ребра не повторяются). Как и в случае гиперграфа, в последующем, множество вершин б.к.р. гиперграфа $H$ будем обозначать также и через $V H$, а семейство его ребер через $\mathscr{E} H$. Если $|V|=n$ и $|\mathscr{E}|=m$, то пару $H$ назовем $(n, m)$-гиперграфом без кратных ребер (б.к.р. $(n, m)$-гиперграфом).

Заметим, что граф является не только специальным случаем гиперграфа, но и специальным случаем б.к.р. гиперграфа. Говорим, что вершина $v \in V$ является инцидентной некоторому ребру $e$ (упорядоченного, б.к.р.) гиперграфа $H$, если $v \in e$. Множество $V^{\prime} \subseteq V$ является множеством смежных вершин (упорядоченного, б.к.р.) гиперграфа, если существует ребро $e$ которое содержит все вершины этого множества, т.е. $V^{\prime} \subseteq$ е. Гиперграф (упорядоченный гиперграф, б.к.р. гиперграф) $H$ называется $k$-полным, $k=1, \ldots,|V|$, если любое подмножество $V^{\prime} \subseteq V,\left|V^{\prime}\right| \leqslant k$, является множеством его смежных вершин. В специальном случае, когда (упорядоченный, б.к.р.) гиперграф $H$ является $|V|$-полным, говорим, также, что он $\infty$-полный.

Предполагаем, что любой (упорядоченный, б.к.р.) гиперграф, появляющийся в последующем, будет помеченным, т.е. на множестве его вершин задан некоторый линейный порядок.

Гиперграф (упорядоченн гиперграф, б.к.р. гиперграф) $H$ есть $T_{0}$-гиперграф (упорядоченный $T_{0}$-гиперграф, б.к.р. $T_{0}$-гиперграф), если для любых $u, v \in V$ существует ребро $e$ в $H$ такое, что $(u \in e \wedge v \notin e) \vee(u \notin e \wedge v \in e)$.

Пусть $H=(V, \mathscr{E}), \mathscr{E}=\left(e_{\lambda}, \lambda \in \Lambda\right)$, - гиперграф. Для любого семейства $\mathscr{A}=$ $\left(\alpha_{\lambda}, \lambda \in \Lambda\right)$ элементов $\alpha_{\lambda}$ из множества $\{0,1\}$ пусть

$$
\sigma_{\mathscr{A}}(H)=\bigcap_{\lambda \in \Lambda} e_{\lambda}^{\alpha_{\lambda}},
$$


где

$$
e^{\alpha}= \begin{cases}e, & \text { если } \alpha=1 \\ V \backslash e, & \text { если } \alpha=0\end{cases}
$$

(эти множества $\sigma_{\mathscr{A}}(H)$ отвечают по смыслу конституентам множеств семейства $\mathscr{E}$ [3]). Ясно, что для любого $v \in V$ существует семейство $\mathscr{A}$ такое, что $v \in \sigma_{\mathscr{A}}(H) \mathrm{K}$ тому же $\sigma_{\mathscr{A}}(H) \cap \sigma_{\mathscr{A}^{\prime}}(H)=\varnothing$ для любых двух различных семейств $\mathscr{A}$ и $\mathscr{A}^{\prime}$. Значит, множества

$$
\sigma(H)=\left\{\sigma_{\mathscr{A}}(H) \mid \mathscr{A} \in\{0,1\}^{\Lambda}, \sigma_{\mathscr{A}}(H) \neq \varnothing\right\}
$$

образуют разбиение множества $V$. Обозначим через $[v]$ элемент $\sigma_{\mathscr{A}} \in \sigma(H)$, для которого $v \in \sigma_{\mathscr{A}}$, а через $\left[e_{\lambda}\right]$ множество $\left\{[v] \mid v \in e_{\lambda}\right\}$. Тогда гиперграф $[H]=([V],[\mathscr{E}])$, где $[V]=\{[v], v \in V\}$, a $[\mathscr{E}]=\left(\left[e_{\lambda}\right], \lambda \in \Lambda\right)$, называется ассоциированным гиперграфом гиперграфа $H$. Аналогично, для любого (б.к.р. гиперграфа) упорядоченного гиперграфа $H$ определяется соответствующий ассоциированный (б.к.р. гиперграф) упорядоченный гиперграф $[H]$.

Теперь вершинам гиперграфов будем приписывать один из двух цветов, скажем, зеленый или красный. Раскраской гиперграфа называется такое приписывание зеленого и красного цвета его вершинам, что любое его непустое ребро содержит хотя бы одну вершину, которой приписан зеленый цвет. Очевидно, что множество всех вершин, ко'торым приписан красный цвет, есть независимое множество вершин данного гиперграфа ([4]), и что любое независимое множество, если всем вершинам, принадлежащим ему, приписать красный цвет, а остальным вершинам зеленый, определяет одну из его раскрасок. Таким же способом, как выше, можно определить понятие раскраски для упорядоченного гиперграфа или б.к.р. гиперграфа. Число всех различных раскрасок (упорядоченного, б.к.р.) гиперграфа $H$ обозначим через $\eta(H)$.

Опишем более подробно те классы Поста $[1,2]$, которые нам потребуются ниже. Приведем сначала следующие классы: $C_{1}-$ класс всех функций алгебры логики; $C_{2}$ - класс всех функций алгебры логики $f$, для которых $f(0, \ldots, 0)=0 ; C_{3}-$ класс всех функций алгебры логики $f$, для которых $f(1, \ldots, 1)=1 ; C_{4}=C_{2} \cap C_{3}$.

Пусть $\mu$ и $i-$ натуральные числа такие, что $\mu \geqslant 2$ и $i \in\{0,1\}$. Функция алгебры логики $f$ от $n$ переменных удовлетворяет условию $a_{i}^{\mu}$, если для любого множества наборов из $f^{-1}(i)$ мощности, не большей $\mu$, существует $j, 1 \leqslant j \leqslant n$, такое, что $j$-я компонента всех этих наборов равна $i$. Тогда $F_{4}^{\mu}-$ класс всех функций алгебры логики, удовлетворяющих условию $a_{0}^{\mu} ; F_{1}^{\mu}=C_{4} \cap F_{4}^{\mu} ; F_{8}^{\mu}-$ класс всех функций алгебры логики, удовлетворяющих условию $a_{1}^{\mu} ; F_{5}^{\mu}=C_{4} \cap F_{8}^{\mu}$.

Функция алгебры логики $f$ от $n$ переменных удовлетворяет условию $a_{i}^{\infty}, i=$ 0,1 , если существует $j, 1 \leqslant j \leqslant n$, такое, что любой набор из множества $f^{-1}(i)$ имеет $j$-ю компоненту, равную $i$. Тогда $F_{4}^{\infty}$ - класс всех функций алгебры логики, удовлетворяющих условию $a_{0}^{\infty} ; F_{1}^{\infty}=C_{4} \cap F_{4}^{\infty} ; F_{8}^{\infty}-$ класс всех функций алгебры логики, удовлетворяющих условию $a_{1}^{\infty} ; F_{5}^{\infty}=C_{4} \cap F_{8}^{\infty}$.

Функция алгебры логики $f$ имеет ранг $k, 0 \leqslant k \leqslant 2^{n}$, если $\left|f^{-1}(1)\right|=k$. Если $\mathscr{P}-$ класс Поста, то множество всех функций от $n$ переменных ранга $k$ из $\mathscr{P}$ обозначим через $[\mathscr{P}]_{k}^{(n)} ;$ множество $[\mathscr{P}]_{k}^{(n)}$ назовем $(n, k)$-сечением класса $\mathscr{P}$.

5 Дискретная математика, т.11 №4 


\section{3. Число функций алгебры логики в классах $\left[F_{8}^{\mu}\right]_{k}^{(n)}$ $\mathbf{и}\left[F_{5}^{\mu}\right]_{k}^{(n)}$}

Целью этого параграфа является нахождение числа $\alpha_{\mu}(k, n)=\left|\left[F_{8}^{\mu}\right]_{k}^{(n)}\right|$.

Обозначим через $\gamma_{\mu}(n, m)$ число всех упорядоченных $\mu$-полных $(n, m)$-гиперграфов, через $\beta_{\mu}(n, m)$ число всех упорядоченных $\mu$-полных $T_{0}$-гиперграфов, у которьх в точности $n$ вершин и $m$ ребер, а через $S(n, k)$ числа Стирлинга второго рода [5]. Два (упорядоченных, б.к.р.) гиперграфа $H$ и $H^{\prime}$ считаем (тождественно) равными (и пишем $H \equiv H^{\prime}$ ), если $V H=V H^{\prime}$ и $\mathscr{E} H=\mathscr{E} H^{\prime}$. Имеет место следующее утверждение.

Лемма 1. Справедливо равенство

$$
\sum_{k=1}^{n} S(n, k) \beta_{\mu}(k, m)=\gamma_{\mu}(n, m)
$$

Доказателъство. Заметим, что для любого (упорядоченного, б.к.р.) гиперграфа $H$ ассоциированный ему (упорядоченный, б.к.р.) гиперграф $[H]$ является (упорядоченным $T_{0}$-гиперграфом, б.к.р. $T_{0}$-гиперграфом) $T_{0}$-гиперграфом. Кроме того, для любого $\mu$-полного (упорядоченного, б.к.р.) гиперграфа $H$ (упорядоченный, б.к.р.) гиперграф $[H]$ является тоже $\mu$-полным, и обратно. Фиксируем некоторое множество $V,|V|=n$. Обозначим класс всех упорядоченных $\mu$-полных $(n, m)$-гиперграфов на множестве $V$ через $\Gamma_{\mu}(n, m)$. Из сказанного выше следует, что все упорядоченные гиперграфы из

$$
\left[\Gamma_{\mu}(n, m)\right]=\left\{[H] \mid H \in \Gamma_{\mu}(n, m)\right\}
$$

являются упорядоченными $\mu$-полными $T_{0}$-гиперграфами. Если $H \equiv H^{\prime}$, где $H, H^{\prime} \in$ $\Gamma_{\mu}(n, m)$, то $[H] \equiv\left[H^{\prime}\right]$, и обратно. Разобьем множество $\left[\Gamma_{\mu}(n, m)\right]$ на непересекающиеся классы

$$
\left[\Gamma_{\mu}(n, m)\right]_{k}, \quad k=1, \ldots, n,
$$

таким способом, что $H \in\left[\Gamma_{\mu}(n, m)\right]$ принадлежит классу $\left[\Gamma_{\mu}(n, m)\right]_{k}$, если $|V[H]|=k$. Далее, разобьем любой класс $\left[\Gamma_{\mu}(n, m)\right]_{k}$ на подклассы

$$
\left[\Gamma_{\mu}(n, m)\right]_{k, l}, \quad l=1, \ldots, l_{k},
$$

таким способом, что $H, H^{\prime} \in\left[\Gamma_{\mu}(n, m)\right]_{k}$ принадлежат одному и тому же подклассу тогда и только тогда, когда $\sigma(H)=\sigma\left(H^{\prime}\right)$ (см. выше определение ассоциированного упорядоченного гиперграфа). Ясно, что $l_{k}$ равно числу всех разбиений $n$-множества $V$ на $k$ блоков, т.е. $l_{k}=S(n, k)$ для любого $k=1, \ldots, n$, и что $\left|\left[\Gamma_{\mu}(n, m)\right]_{k, l}\right|=$ $\beta_{\mu}(k, m)$ для любых $k=1, \ldots, n$ и $l=1, \ldots, l_{k}$. Отсюда следует, что

$$
\gamma_{\mu}(n, m)=\left|\Gamma_{\mu}(n, m)\right|=\sum_{k=1}^{n} \sum_{l=1}^{S(n, k)}\left|\left[\Gamma_{\mu}(n, m)\right]_{k, l}\right|=\sum_{k=1}^{n} S(n, k) \beta_{\mu}(k, m) .
$$

Лемма 1 доказана.

Зафиксируем некоторое множество $V,|V|=n$. Через $\mathscr{H}_{n} \leqslant \mu, \mu \geqslant 2$, обозначим класс всех б.к.р. гиперграфов $\left(V, \mathscr{E}^{\prime}\right)$ таких, что $\varnothing \notin \mathscr{E}^{\prime}$ и $|e| \leqslant \mu$ для любого $e \in \mathscr{E}^{\prime}$ (случай $\mathscr{E}^{\prime}=\varnothing$ также учитывается). 
Лемма 2. Справедливо равенство

$$
\gamma_{\mu}(n, m)=\sum_{H \in \mathscr{H}_{n}^{\leqslant \mu}}(-1)^{|\mathscr{E} H|} \eta^{m}(H)
$$

Доказателъство. Обозначим через $\mathscr{H}_{n, m}$ класс всех упорядоченных $(n, m)$-гиперграфов с множеством вершин $V$. Пусть $V_{1}, \ldots, V_{k}$ - различные непустые подмножества множества $V$ такие, что $\left|V_{i}\right| \leqslant \mu$ для любого $i=1, \ldots, k$. Мы говорим, что упорядоченный гиперграф $H^{\prime} \in \mathscr{H}_{n, m}$ удовлетворяет условиям $p\left(V_{1}\right), \ldots, p\left(V_{k}\right)$ тогда и только тогда, когда множества $V_{1}, \ldots, V_{k}$ не являются множествами смежных вершин в $H^{\prime}$. Подсчитаем, сколькими способами можно выбрать подмножества $e_{1}, \ldots, e_{m}$ множества $V$ так, чтобы упорядоченный гиперграф $\left(e_{1}, \ldots, e_{m}\right)$ удовлетворял условиям $p\left(V_{1}\right), \ldots, p\left(V_{k}\right)$. Зафиксируем некоторое $j, 1 \leqslant j \leqslant m$, и подсчитаем, сколькими способами мы можем выбрать множество $e_{j}$ так, что $V_{i} \nsubseteq e_{j}$ для всех $i=1, \ldots, k$. Предположим, что у нас есть один такой (правильный) выбор для ребра $e_{j}$. Рассмотрим также б.к.р. гиперграф $H=\left(V,\left\{V_{1}, \ldots, V_{k}\right\}\right)$. Припишем вершине из $H$ красный цвет, если она принадлежит множеству $e_{j}$, и зеленый цвет в противном случае. Ясно, что любой правильный выбор для ребра $e_{j}$ должен быть таким, что ни одно ребро б.к.р. гиперграфа $H$ не содержит вершин, окрашенных только в красный цвет, т.е. любой такой выбор определяет одну раскраску б.к.р. гиперграфа $H$. Обратное также имеет место, а именно, любая раскраска б.к.р. гиперграфа $H$ определяет, естественным способом, правильный выбор для ребра $e_{j}$. Следовательно, существует $\eta(H)$ возможных выборов для ребра $e_{j}$. Поскольку ребра $e_{1}, \ldots, e_{m}$ выбираются независимо друг от друга, существует $\eta^{m}(H)$ искомых упорядоченных гиперграфов. Б.к.р. гиперграф $H$ может быть любым б.к.р. гиперграфом из множества $\mathscr{H}_{n}^{\leqslant \mu}$ с $k$ ребрами. Число всех упорядоченных гиперграфов из $\mathscr{H}_{n, m}$, которые не удовлетворяют ни одному свойству $p\left(V^{\prime}\right)$, где $V^{\prime}$ - непустое подмножество множества $V$ и $\left|V^{\prime}\right| \leqslant \mu$, равно числу $\gamma_{\mu}(n, m)$ всех упорядоченных $\mu$-полных $(n, m)$-гиперграфов. Заметим, что $(V, \varnothing) \in \mathscr{H}_{n}^{\leqslant \mu}$ и число $\eta^{m}[(V, \varnothing)]=2^{n m}$ равно мощности множества $\mathscr{H}_{n, m}$. Используя далее принцип включения-исключения, получаем утверждение леммы 2.

Обозначим через $\mathscr{H}_{n}^{=\mu}, \mu \geqslant 2$, класс всех б.к.р. гиперграфов с $n$ вершинами, у которых каждое ребро содержит точно $\min \{n, \mu\}$ вершин (здесь, неявно, предполагаем, что у всех гиперграфов данного класса одно и то же множество вершин мощности $n$ ). В соответствии с этим определением мы будем считать, что $\mathscr{H}_{n}^{=\infty}=\mathscr{H}_{n}^{=n}=\left\{0_{n}, 1_{n}\right\}$, где $0_{n}$-- б.к.р. гиперграф (с $n$ вершинами) и пустым множеством ребер, и $1_{n}$ - б.к.р. гиперграф, у которого единственное ребро содержит все $n$ его вершин.

Покажем теперь, что формулу из леммы 2 можно значительно упростить, редуцируя класс $\mathscr{H}_{n} \leqslant \mu$ (над которым выполняется суммирование) до класса $\mathscr{H}_{n}^{=\mu}$.

Пусть $H=(V, \mathscr{E})$ - некоторый б.к.р. гиперграф. Выделим в $\mathscr{E}$ все минимальные ребра по отношению к включению $\subseteq$ и обозначим это множество через $S(\mathscr{E})$, т.е. $e \in S(\mathscr{E})$, если не существует ребро $e^{\prime} \in S(\mathscr{E})$ такое, что $e^{\prime} \subseteq e$. Пусть $H$ и $H^{\prime}-$ два б.к.р. гиперграфа с одним и тем же множеством вершин. Будем писать $H \sim H^{\prime}$, если $S(\mathscr{E} H)=S\left(\mathscr{E} H^{\prime}\right)$. Легко удостовериться, что отношение $\sim$ является отношением эквивалентности и из соотношения $H \sim H^{\prime}$ следует, что $\eta(H)=\eta\left(H^{\prime}\right)$. Имеет место следующее утверждение. 
Лемма 3. Справедливо равенство

$$
\gamma_{\mu}(n, m)=\sum_{H \in \mathscr{H}_{n}^{=\mu}}(-1)^{|\mathscr{E} H|} \eta^{m}(H)
$$

Доказательство. Зафиксируем некоторое множество $V,|V|=n$. Рассмотрим множество б.к.р. гиперграфов $\mathscr{H}_{n} \leqslant \mu$ и на нем отношение эквивалентности $\sim$. Классы эквивалентности $\left(\mathscr{H}_{n}^{\leqslant \mu}\right)_{k}, k=1, \ldots, l_{0}$, отношения $\sim$ определяют одно разбиение множества $\mathscr{H}_{n}^{\leqslant \mu}$. В любом классе $\left(\mathscr{H}_{n}^{\leqslant \mu}\right)_{k}, k=1, \ldots, l_{0}$, существует единственный б.к.р. гиперграф $H$ такой, что $\mathscr{E} H \subseteq \mathscr{E}^{\prime}$ для любого $H^{\prime} \in\left(\mathscr{H}_{n}^{\leqslant \mu}\right)_{k}$; про такой б.к.р. гиперграф $H$ говорим, что он порождает класс $\left(\mathscr{H}_{n}^{\leqslant \mu}\right)_{k}$. Ясно, что любой $H \in \mathscr{H}_{n}=\mu$ порождает некоторый подкласс, который в качестве единственного элемента имеет гиперграф $H$. Если $H \in \mathscr{H}_{n}^{\leqslant \mu} \backslash \mathscr{H}_{n}^{=\mu}$ и $H$ порождает некоторый подкласс, тогда множество

$$
\Delta(H)=\left\{e \subseteq V \mid \text { существует } e^{\prime} \in \mathscr{E} H, e^{\prime} \subseteq e\right\}
$$

не является пустым. Поскольку из соотношения $H \sim H^{\prime}$ следует, что $\eta(H)=\eta\left(H^{\prime}\right)$, получаем, что

$$
\sum_{H^{\prime} \in\left(\mathscr{H}_{n}^{\leq \mu}\right)_{i(H)}}(-1)^{\left|\mathscr{E} H^{\prime}\right|} \eta^{m a}\left(H^{\prime}\right)=(-1)^{|\mathscr{E} H|} \eta^{m}(H) \sum_{\Delta^{\prime} \subseteq \Delta(H)}(-1)^{\left|\Delta^{\prime}\right|}=0,
$$

где $\left(\mathscr{H}_{n}^{\leqslant \mu}\right)_{i(H)}-$ подкласс, порожденный гиперграфом $H$. Отсюда, в силу леммы 2 , следует утверждение леммы 3.

Возьмем $f \in\left[F_{8}^{\mu}\right]_{k}^{(n)}$ и рассмотрим множество мощности $k$ всех двоичных наборов длины $n$, на которых $f$ принимает значение 1. Зафиксируем некоторый линейный порядок на этом множестве. Обозначим через $S_{f}$ множество всех перестановок этого множества. Ясно, что $\left|S_{f}\right|=k$ !. Теперь возьмем некоторое $s \in S_{f}$ и в соответствии с этой перестановкой $s$ выпишем один над другим эти наборы. Полученную таким способом матрицу можно интерпретировать как матрицу инцидентности некоторого упорядоченного $\mu$-полного $(k, n)$-гиперграфа $H_{f}^{s}$. Имеет место следующее утверждение.

Лемма 4. Справедливо равенство

$$
\alpha_{\mu}(k, n)=\frac{1}{k !} \beta_{\mu}(k, n) .
$$

Доказателъство. Для любой функции $f \in\left[F_{8}^{\mu}\right]_{k}^{(n)}$ и любой перестановки $s \in S_{f}$ соответствующий упорядоченный $(k, n)$-гиперграф $H_{f}^{s}$ является $\mu$-полным $T_{0}$-гиперграфом. И наоборот, для любого упорядоченного $\mu$-полного $T_{0}$-гиперграфа $H$, у которого $k$ вершин и $n$ ребер, существуют такие $f \in\left[F_{8}^{\mu}\right]_{k}^{(n)}$ и $s \in S_{f}$, что $H=H_{f}^{s}$.

Пусть $s(n, k)$ - числа Стирлинга первого рода [5]. Суммируем результаты предыдущих лемм и докажем основной результат нашей работы.

Теорема 1. Справедливо равенство

$$
\alpha_{\mu}(n, m)=\frac{1}{n !} \sum_{i=1}^{n} s(n, i) \sum_{H \in \mathscr{H}_{i}=\mu}(-1)^{|\mathscr{E} H|} \eta^{m}(H) .
$$


Доказательство. Обращением Стирлинга [5] из формулы в лемме 1 получаем равенство

$$
\beta_{\mu}(n, m)=\sum_{i=1}^{n} s(n, i) \gamma_{\mu}(i, m)
$$

Тогда из леммы 3 и леммы 4 вытекает утверждение теоремы 1 .

Теорема 1 сводит нашу проблему к определению числа различных раскрасок произвольного б.к.р. гиперграфа $H$ из класса $\mathscr{H}_{i}^{=\mu}, i=1,2, \ldots$ Поэтому очень важно найти метод для определения этого числа для произвольного б.к.р. гиперграфа $H=(V, \mathscr{E})$, у которого нет пустого ребра (ясно, что наличие пустого ребра у б.к.р. гиперграфа не существенно - число его раскрасок не зависит от этого). Возьмем произвольную вершину $v$ б.к.р. гиперграфа $H$, и пусть $\mathscr{E}_{v}-$ множество всех ребер данного б.к.р. гиперграфа, инцидентных $v$. Введем обозначения

$$
\mathscr{E}_{v}^{1}=\left(\left\{e \backslash\{v\} \mid e \in \mathscr{E}_{v}\right\} \backslash\{\varnothing\}\right) \cup\left(\mathscr{E} \backslash \mathscr{E}_{v}\right), \quad \mathscr{E}_{v}^{2}=\mathscr{E} \backslash \mathscr{E}_{v}
$$

Рассмотрим пары $H_{v}^{1}=\left(V \backslash\{v\}, \mathscr{E}_{v}^{1}\right)$ и $H_{v}^{2}=\left(V \backslash\{v\}, \mathscr{E}_{v}^{2}\right)$. Если $|V| \geqslant 2$, то пары $H_{v}^{1}$ и $H_{v}^{2}$ являются б.к.р. гиперграфами. Введем формально символ $0_{0}$ полагая, что $0_{0}=(\varnothing, \varnothing)$. Расширим область определения функции $\eta$ полагая, что $\eta\left(0_{0}\right)=1$. Для любого $v \in V$ определим $\nu_{H}(v)$ таким способом, что $\nu_{H}(v)=0$, если существует ребро, которое содержит только вершину $v$, и $\nu_{H}(v)=1$ в противном случае.

Предложение 1. Пусть $H=(V, \mathscr{E})$ - б.к.p. гиперграф без пустого ребра $u v-$ некоторая его вершина. Тогда

$$
\eta(H)=\nu_{H}(v) \eta\left(H_{v}^{1}\right)+\eta\left(H_{v}^{2}\right)
$$

Ограничимся теперь случаями, когда $\mu=2$ и $\mu=\infty$.

Предположим сначала, что $\mu=2$. Обозначим через $\mathscr{G}_{i}$ множество всех графов, у которых $i$ вершин. Заметим, что $\mathscr{G}_{i}=\mathscr{H}_{i}{ }^{=2}$, если $i \geqslant 2$, и по определению $\mathscr{H}_{1}^{=2}=$ $\mathscr{H}_{1}^{=1}=\left\{0_{1}, 1_{1}\right\}$. Согласно теореме 1 имеет место следующее утверждение.

Теорема 2. Справедливо равенство

$$
\alpha_{2}(n, m)=\frac{1}{n !}\left((-1)^{n}(n-1) !+\sum_{i=1}^{n} s(n, i) \sum_{G \in \mathscr{G}_{i}}(-1)^{|\mathscr{E} G|} \eta^{m}(G)\right)
$$

В случае графов предложение 1 можно переформулировать следующим образом. Пусть $G$ - некоторый граф (заметим еще раз, что граф является специальным случаем б.к.р. гиперграфа, так что символы $G_{v}^{1}$ и $G_{v}^{2}$ имеют такой же смысл, как и выше). Обозначим через $\hat{G}_{v}^{1}$ следующую пару. Если $|V|=1$, то $\hat{G}_{v}^{1}=0_{0}$, а если $|V| \geqslant 2$, то $\hat{G}_{v}^{1}$ является гиперграфом, полученным из $G$, когда $v$ и все ему смежные вершины, а также все ребра, которые инцидентны этим вершинам, отбрасываются.

Предложение 2. Пусть $G$ - некоторый граф $u v$-некоторая его вериина. Тогда имеет место равенство

$$
\eta(G)=\eta\left(\hat{G}_{v}^{1}\right)+\eta\left(G_{v}^{2}\right)
$$




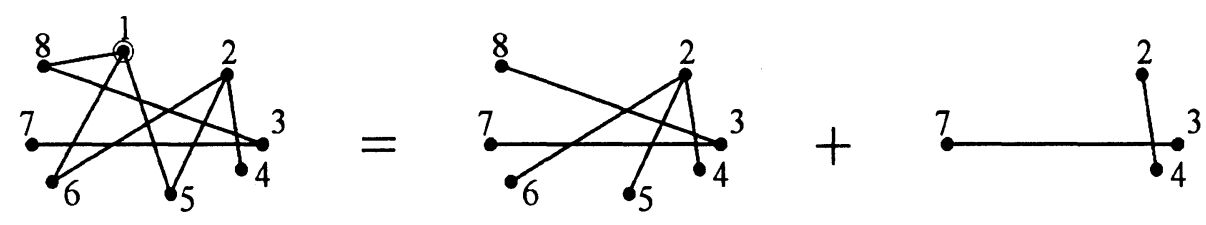

Pис. 1.

Доказательство. Из предложения 1 получаем, что

$$
\eta(G)=\nu_{G}(v) \eta\left(G_{v}^{1}\right)+\eta\left(G_{v}^{2}\right) .
$$

Заметим, что вершины, которые покрыты одноэлементным ребром должны быть окрашены в зеленый цвег, и что тогда их можно отбросить вместе со всеми им инцидентными ребрами, не меняя числа возможных раскрасок, т.е. $\eta\left(G_{v}^{1}\right)=\eta\left(\hat{G}_{v}^{1}\right)$. Также ясно, что $\nu_{G}\left(v^{\prime}\right)=1$ для любого $v^{\prime} \in V G$. Значит, вместо вышеприведенного равенства получаем равенство

$$
\eta(G)=\eta\left(\hat{G}_{v}^{1}\right)+\eta\left(G_{v}^{2}\right)
$$

которое и требовалось доказать.

Пользуясь этим предложением, легко вычислить $\eta(G)$ для некоторых специальных классов графов $G$.

Пример 1. Пусть $K_{n}$ и $K_{m, n}-$ соответственно полный граф, у которого $n$ вершин, и полный двудольный граф с блоками из $m$ и $n$ вершин. Тогда

$$
\eta\left(K_{n}\right)=n+1, \quad \eta\left(K_{m, n}\right)=2^{m}+2^{n}-1 .
$$

Если $P_{n}-$ путь, у которого $n$ вершин, то получаем числа Фибоначчи

$$
\eta\left(P_{n}\right)=\eta\left(P_{n-1}\right)+\eta\left(P_{n-2}\right), \quad n=2,3, \ldots, \quad \eta\left(P_{0}\right)=1, \quad \eta\left(P_{1}\right)=2 .
$$

Если $Z_{n}-n$-цикл, то получаем, что

$$
\eta\left(Z_{n}\right)=\eta\left(P_{n-1}\right)+\eta\left(P_{n-3}\right), \quad n=3,4, \ldots
$$

Лемма 5. Если $C_{1}, C_{2}, \ldots, C_{i}$ - все компоненты связности (махсимальные связнъе подграфы) графа $G$, по

$$
\eta(G)=\eta\left(C_{1}\right) \eta\left(C_{2}\right) \ldots \eta\left(C_{i}\right) .
$$

Доказательство этой леммы очевидно.

Пример 2. Теперь мы можем легко вычислить $\eta(G)$, например, для графа $G$, приведенного на рис. 1 (разбиение начинается с вершины, отмеченной единицей).

Получаем, что

$$
\eta(G)=\eta\left(P_{3}\right) \eta\left(K_{1,3}\right)+\eta\left(P_{2}\right) \eta\left(P_{2}\right)=5 \cdot 9+3 \cdot 3=54 .
$$




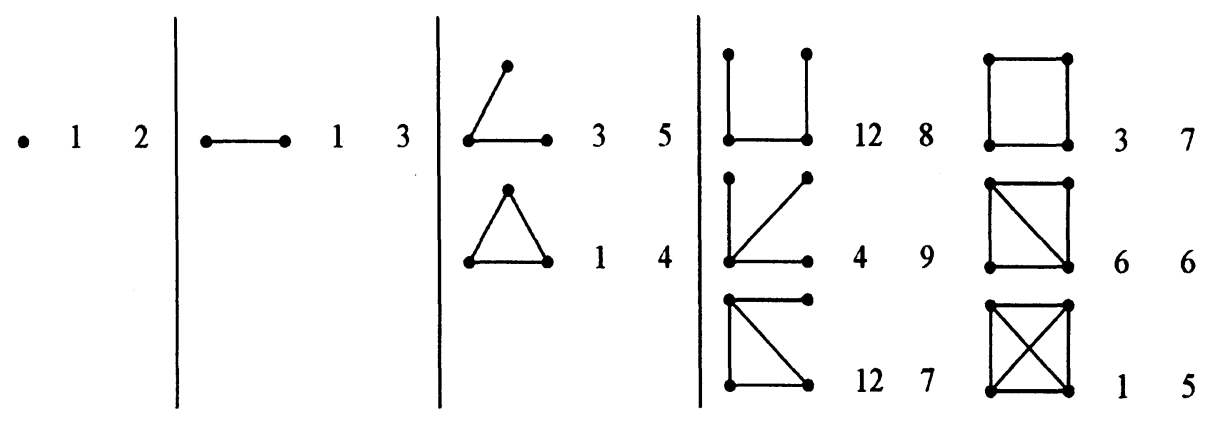

Рис. 2.

Пусть $B_{n}\left(k_{1}, k_{2}, \ldots, k_{n}\right)$ - число разбиений $n$-множества на $k_{1}+k_{2}+\ldots+k_{n}$ частей, среди которых имеется в точности $k_{i}$ подмножеств с $i$ элементами, $1 \leqslant i \leqslant n$. Тогда многочлены

$$
P_{n}\left(x_{1}, x_{2}, \ldots, x_{n}\right)=\sum_{\left(k_{1}, k_{2}, \ldots, k_{n}\right)} B_{n}\left(k_{1}, k_{2}, \ldots, k_{n}\right) x_{1}^{k_{1}} x_{2}^{k_{2}} \ldots x_{n}^{k_{n}},
$$

где $k_{1}+2 k_{2}+\ldots+n k_{n}=n\left(k_{i} \geqslant 0, i=1, \ldots, n\right)$, называются полиномами Белла [6]. На основании леммы 5 , теорему 2 можно переформулировать следующим способом.

Теорема 3. Пусть $\mathscr{G}_{j}^{c}-$ множество всех свлзных графов, у которых $j$ вершин. Тогда

$$
\alpha_{2}(n, m)=\frac{1}{n !}\left((-1)^{n}(n-1) !+\sum_{i=1}^{n} s(n, i) P_{i}\left(\gamma_{2}^{c}(1, m), \gamma_{2}^{c}(2, m), \ldots, \gamma_{2}^{c}(i, m)\right)\right)
$$

гдe

$$
\gamma_{2}^{c}(j, m)=\sum_{G \in \mathscr{G}_{j}^{c}}(-1)^{|\mathscr{E}(G)|} \eta^{m}(G), \quad j=1,2, \ldots
$$

Пример 3. Нетрудно перечислить все неизоморфные связные графы, у которых не более четырех вершин (см., например, каталог графов в [7]). На рис. 2 в первом столбце указано число изоморфных копий данного графа, а во втором число всех различных их раскрасок.

Справедливы равенства

$$
\begin{aligned}
& \gamma_{2}^{c}(1, m)=2^{m}, \\
& \gamma_{2}^{c}(2, m)=-3^{m} \\
& \gamma_{2}^{c}(3, m)=3 \cdot 5^{m}-4^{m}, \\
& \gamma_{2}^{c}(4, m)=-4 \cdot 9^{m}-12 \cdot 8^{m}+15 \cdot 7^{m}-6 \cdot 6^{m}+5^{m} .
\end{aligned}
$$

Заменой $x_{i}$ на $\gamma_{2}^{c}(i, m)$ в многочленах Белла (список многочленов Белла дан в [6]) и с учетом теоремы 3 получаем формулы для $\alpha_{2}(n, m), 1 \leqslant n \leqslant 4$, которые приведены в последнем параграфе настоящей статьи. 
Рассмотрим случай $\mu=\infty$, т.е. определим число

$$
\alpha_{\infty}(n, m)=\left|\left[F_{8}^{\infty}\right]_{n}^{(m)}\right|
$$

Теорема 4. Справедливо равенство

$$
\alpha_{\infty}(n, m)=\sum_{j=1}^{m}(-1)^{j+1}\left(\begin{array}{c}
m \\
j
\end{array}\right)\left(\begin{array}{c}
2^{m-j} \\
n
\end{array}\right)
$$

Доказательство. Поскольку $\mathscr{H}_{i}^{=i}=\left\{0_{i}, 1_{i}\right\}, \eta\left(0_{i}\right)=2^{i}, \eta\left(1_{i}\right)=2^{i}-1$, из теоремы 1 и из определения чисел Стирлинга первого рода [5] следует, что

$$
\begin{aligned}
\alpha_{\infty}(n, m) & =\frac{1}{n !} \sum_{i=1}^{n} s(n, i) \sum_{H \in \mathscr{H}_{i}=i}(-1)^{|\mathscr{E} H|} \eta^{m}(H) \\
& =\frac{1}{n !} \sum_{i=1}^{n} s(n, i)\left(2^{i m}-\left(2^{i}-1\right)^{m}\right) \\
& =\frac{1}{n !} \sum_{i=1}^{n} s(n, i) \sum_{j=1}^{m}(-1)^{j+1}\left(\begin{array}{c}
m \\
j
\end{array}\right)\left(2^{m-j}\right)^{i} \\
& =\frac{1}{n !} \sum_{j=1}^{m}(-1)^{j+1}\left(\begin{array}{c}
m \\
j
\end{array}\right) \sum_{i=1}^{n} s(n, i)\left(2^{m-j}\right)^{i} \\
& =\frac{1}{n !} \sum_{j=1}^{m}(-1)^{j+1}\left(\begin{array}{c}
m \\
j
\end{array}\right)\left[2^{m-j}\right]_{n}=\sum_{j=1}^{m}(-1)^{j+1}\left(\begin{array}{c}
m \\
j
\end{array}\right)\left(\begin{array}{c}
2^{m-j} \\
n
\end{array}\right)
\end{aligned}
$$

где $[n]_{m}=n(n-1) \ldots(n-m+1)$. Конечно, эту теорему можно также доказать и непосредственно (без использования теоремы 1 ).

Тогда общее число функций из класса $\left[F_{8}^{\infty}\right]^{(m)}$ (произвольного ранга) есть

$$
\sum_{j=1}^{m}(-1)^{j+1}\left(\begin{array}{c}
m \\
j
\end{array}\right) 2^{2^{m-j}}
$$

Теперь мы также можем определить мощность классов $\left[F_{5}^{\mu}\right]_{k}^{(n)}$. Пусть

$$
\alpha_{\mu}^{(1)}(n, m)=\left|\left[F_{5}^{\mu}\right]_{n}^{(m)}\right|, \quad \alpha_{\mu}^{(0)}(n, m)=\left|\left[F_{8}^{\mu}\right]_{n}^{(m)} \backslash\left[F_{5}^{\mu}\right]_{n}^{(m)}\right| .
$$

Формально введем $\mathscr{H}_{0}^{=0}=\left\{0_{0}, 1_{0}\right\}$, полагая, что $\left|\mathscr{E}_{0}\right|=0,\left|\mathscr{E} 1_{0}\right|=1, \eta\left(0_{0}\right)=1$ и $\eta\left(1_{0}\right)=0$.

\section{Теорема 5. Справедливъ равенства}

$$
\alpha_{\mu}^{(1)}(n, m)=\frac{1}{(n-1) !} \sum_{i=1}^{n} s(n, i) \sum_{H \in \mathscr{H}_{i-1}^{=\mu}}(-1)^{|\mathscr{E} H|} \eta^{m}(H), \quad n=1,2, \ldots
$$

Доказательство. Поскольку

$$
\alpha_{\mu}(n, m)=\alpha_{\mu}^{(1)}(n, m)+\alpha_{\mu}^{(0)}(n, m), \quad \alpha_{\mu}^{(1)}(n, m)=\alpha_{\mu}^{(0)}(n-1, m),
$$


для любого $n=1,2, \ldots$ и $\alpha_{\mu}^{(0)}(0, m)=1$, справедливо рекуррентное соотношение

$$
\alpha_{\mu}^{(1)}(n, m)=\alpha_{\mu}(n-1, \dot{m})-\alpha_{\mu}^{(1)}(n-1, m), \quad \alpha_{\mu}^{(1)}(0, m)=0 .
$$

Из этой формулы получаем, что

$$
\alpha_{\mu}^{(1)}(n, m)=\sum_{j=1}^{n}(-1)^{j+1} \alpha_{\mu}(n-j, m), \quad \alpha_{\mu}(0, m)=1 .
$$

Отсюда, пользуясь теоремой 1 и рекуррентным соотношением

$$
s(n, i)=\sum_{j=1}^{n}(-1)^{j+1}[n-1]_{j} s(n-j, i-1)
$$

для чисел Стирлинга первого рода [5], получаем утверждение теоремы 5.

Формула теоремы 5 справедлива также в случае, когда $\mu$ заменяется на $\infty$ :

$$
\alpha_{\infty}^{(1)}(n, m)=\sum_{j=1}^{m}(-1)^{j+1}\left(\begin{array}{c}
m \\
j
\end{array}\right)\left(\begin{array}{c}
2^{m-j}-1 \\
n-1
\end{array}\right) .
$$

\section{4. Список производящих функций}

Используя формулы из теоремы 1 и предложения 1, 3. Максимович (Металлурготехнологический факультет Белградского университета) разработал компьютерную программу для порождения чисел $\left|\left[F_{8}^{\mu}\right]_{n}^{(m)}\right|=\alpha_{\mu}(n, m)$ в случае небольших значений $\mu$ и $n$ и любого $m$. Результаты вычислений приведены ниже.

$$
\begin{aligned}
& \alpha_{2}(1, m)=(1 !)^{-1}\left(2^{m}-1\right) \text {, } \\
& \alpha_{2}(2, m)=(2 !)^{-1}\left(4^{m}-3^{m}-2^{m}+1\right) \text {, } \\
& \alpha_{2}(3, m)=(3 !)^{-1}\left(8^{m}-3 \cdot 6^{m}+3 \cdot 5^{m}-4 \cdot 4^{m}+3 \cdot 3^{m}+2 \cdot 2^{m}-2\right) \text {, } \\
& \alpha_{2}(4, m)=(4 !)^{-1}\left(16^{m}-6 \cdot 12^{m}+12 \cdot 10^{m}-1 \cdot 9^{m}-22 \cdot 8^{m}+15 \cdot 7^{m}+12 \cdot 6^{m}\right. \\
& \left.-17 \cdot 5^{m}+17 \cdot 4^{m}-11 \cdot 3^{m}-6 \cdot 2^{m}+6\right) \text {, } \\
& \alpha_{2}(5, m)=(5 !)^{-1}\left(32^{m}-10 \cdot 24^{m}+30 \cdot 20^{m}-5 \cdot 18^{m}+5 \cdot 17^{m}-80 \cdot 16^{m}\right. \\
& -30 \cdot 15^{m}+135 \cdot 14^{m}+30 \cdot 13^{m}-80 \cdot 12^{m}-2 \cdot 11^{m} \\
& +10 \cdot 10^{m}-100 \cdot 9^{m}+240 \cdot 8^{m}-160 \cdot 7^{m}-44 \cdot 6^{m}+95 \cdot 5^{m} \\
& \left.-85 \cdot 4^{m}+50 \cdot 3^{m}+24 \cdot 2^{m}-24\right) \text {, } \\
& \alpha_{2}(6, m)=(6 !)^{-1}\left(64^{m}-15 \cdot 48^{m}+60 \cdot 40^{m}-15 \cdot 36^{m}+30 \cdot 34^{m}-6 \cdot 33^{m}\right. \\
& -215 \cdot 32^{m}-180 \cdot 30^{m}+585 \cdot 28^{m}+45 \cdot 27^{m}+60 \cdot 26^{m}+150 \cdot 25^{m} \\
& -510 \cdot 24^{m}-360 \cdot 23^{m}+168 \cdot 22^{m}-585 \cdot 21^{m}+795 \cdot 20^{m}+1665 \cdot 19^{m} \\
& -1890 \cdot 18^{m}-2175 \cdot 17^{m}+3305 \cdot 16^{m}+1775 \cdot 15^{m}-3795 \cdot 14^{m} \\
& -870 \cdot 13^{m}+3123 \cdot 12^{m}-1075 \cdot 11^{m}-495 \cdot 10^{m}+1460 \cdot 9^{m} \\
& -2245 \cdot 8^{m}+1424 \cdot 7^{m}+150 \cdot 6^{m}-590 \cdot 5^{m}+499 \cdot 4^{m} \\
& \left.-274 \cdot 3^{m}-120 \cdot 2^{m}+120\right) \text {, }
\end{aligned}
$$




$$
\begin{aligned}
& \alpha_{3}(4, m)=(4 !)^{-1}\left(16^{m}-4 \cdot 14^{m}+6 \cdot 13^{m}-4 \cdot 12^{m}\right. \\
& \left.+11^{m}-6 \cdot 8^{m}+6 \cdot 7^{m}+11 \cdot 4^{m}-11 \cdot 3^{m}-6 \cdot 2^{m}+6\right), \\
& \alpha_{3}(5, m)=(5 !)^{-1}\left(32^{m}-10 \cdot 28^{m}+30 \cdot 26^{m}+5 \cdot 25^{m}\right. \\
& -80 \cdot 24^{m}+45 \cdot 23^{m}+105 \cdot 22^{m}-217 \cdot 21^{m}+205 \cdot 20^{m}-120 \cdot 19^{m} \\
& +45 \cdot 18^{m}-10 \cdot 17^{m}-9 \cdot 16^{m}+40 \cdot 14^{m}-60 \cdot 13^{m}+40 \cdot 12^{m} \\
& \left.-10 \cdot 11^{m}+35 \cdot 8^{m}-35 \cdot 7^{m}-50 \cdot 4^{m}+50 \cdot 3^{m}-24 \cdot 2^{m}+24\right) \text {, } \\
& \alpha_{3}(6, m)=(6 !)^{-1}\left(64^{m}-20 \cdot 56^{m}+90 \cdot 52^{m}+30 \cdot 50^{m}+25 \cdot 49^{m}-420 \cdot 48^{m}\right. \\
& -180 \cdot 47^{m}+450 \cdot 46^{m}+60 \cdot 45^{m}+615 \cdot 44^{m}+1683 \cdot 43^{m} \\
& -3252 \cdot 42^{m}-6030 \cdot 41^{m}+8520 \cdot 40^{m}+10560 \cdot 39^{m}-15849 \cdot 38^{m} \\
& -13005 \cdot 37^{m}+26410 \cdot 36^{m}+10655 \cdot 35^{m}-50385 \cdot 34^{m}+33390 \cdot 33^{m} \\
& +29480 \cdot 32^{m}-82010 \cdot 31^{m}+91215 \cdot 30^{m}-67380 \cdot 29^{m}+36870 \cdot 28^{m} \\
& -15249 \cdot 27^{m}+4380 \cdot 26^{m}-1215 \cdot 25^{m}+1390 \cdot 24^{m}-695 \cdot 23^{m} \\
& -1574 \cdot 22^{m}+3255 \cdot 21^{m}-3075 \cdot 20^{m}+1800 \cdot 19^{m}-675 \cdot 18^{m} \\
& +150 \cdot 17^{m}+70 \cdot 16^{m}-340 \cdot 14^{m}+510 \cdot 13^{m}-340 \cdot 12^{m}+85 \cdot 11^{m} \\
& \left.-225 \cdot 8^{m}+225 \cdot 7^{m}+274 \cdot 4^{m}-274 \cdot 3^{m}-120 \cdot 2^{m}+120\right) \text {, } \\
& \alpha_{4}(5, m)=(5 !)^{-1}\left(32^{m}-5 \cdot 30^{m}+10 \cdot 29^{m}-10 \cdot 28^{m}+5 \cdot 27^{m}-26^{m}-10 \cdot 16^{m}\right. \\
& \left.+10 \cdot 15^{m}+35 \cdot 8^{m}-35 \cdot 7^{m}-50 \cdot 4^{m}+50 \cdot 3^{m}+24 \cdot 2^{m}-24\right) \text {, } \\
& \alpha_{4}(6, m)=(6 !)^{-1}\left(64^{m}-15 \cdot 60^{m}+60 \cdot 58^{m}+25 \cdot 57^{m}-240 \cdot 56^{m}+45 \cdot 55^{m}+705 \cdot 54^{m}\right. \\
& -987 \cdot 53^{m}-351 \cdot 52^{m}+3040 \cdot 51^{m}-5445 \cdot 50^{m}+6105 \cdot 49^{m} \\
& -4939 \cdot 48^{m}+2997 \cdot 47^{m}-1365 \cdot 46^{m}+455 \cdot 45^{m}-105 \cdot 44^{m} \\
& +15 \cdot 43^{m}-42^{m}-15 \cdot 32^{m}+75 \cdot 30^{m}-150 \cdot 29^{m}+150 \cdot 28^{m} \\
& -75 \cdot 27^{m}+15 \cdot 26^{m}+85 \cdot 16^{m}-85 \cdot 15^{m}-225 \cdot 8^{m}+225 \cdot 7^{m} \\
& \left.+274 \cdot 4^{m}-274 \cdot 3^{m}-120 \cdot 2^{m}+120\right) \text {. }
\end{aligned}
$$

\section{Список литературы}

1. Post E., Two-valued iterative systems. Ann. Math. Studies (1941) 5.

2. Яблонский С.В., Гаврилов Г.П., Кудрявцев В.Б., Фуякции алгебры логихи и хлассы Поста. Наука, Москва, 1966.

3. Куратовский К., Мостовский А., Теория множеств. Мир, Москва, 1970.

4. Емеличев В.А., Мельников О.И., Сарванов В.И., Тышкевич Р.И., Лехции по теории графов. Наука, Москва, 1990.

5. Айгнер М., Комбинаторная теория. Мир, Москва, 1982.

6. Риордан Дж., Комбинаторные тождества. Наука, Москва, 1982.

7. Харари Ф., Теория графов. Мир, Москва, 1973.

Статья поступила 12.11.1998. 